\title{
Método acelerado de processamento de presunto cru
}

\author{
Accelerated method of dry-cured ham processing
}

\author{
Walter BERGAMIN FILHO ${ }^{1}$, Marcela de Rezende COSTA ${ }^{1}$, \\ Pedro Eduardo de FELÍCIO ${ }^{1}$, Expedito Tadeu Facco SILVEIRA
}

\section{Resumo}

Nos métodos tradicionais de elaboração de presunto cru usa-se o pernil suíno inteiro, diferentemente da metodologia proposta neste trabalho, que combinou desossa, adição de transglutaminase, massageamento e moldagem das peças previamente à secagem e maturação, objetivando reduzir o tempo de processamento do produto. Avaliaram-se os efeitos de dois teores de $\mathrm{NaCl}$ adicionados (T1 - 3,5\% e T2 - 5\%), sobre características físico-químicas e microbiológicas dos presuntos crus ao longo do processo, além da avaliação sensorial dos produtos finais. Os presuntos crus obtidos atenderam aos padrões físico-químicos e microbiológicos determinados na legislação brasileira e não foram encontradas diferenças significativas $(\mathrm{p}<0,05)$ entre os tratamentos quanto aos parâmetros avaliados no decorrer do processo e no produto final, com exceção da perda de peso, que foi maior em T1 (39,74 $\pm 4,02 \%)$ do que em T2 (37,22 $\pm 2,96 \%)$. Os presuntos crus desenvolvidos apresentaram formato e espessura apropriados para o fatiamento, excelente aparência, aroma característico e um sabor considerado muito próximo ao dos presuntos crus tradicionais comumente encontrados no mercado brasileiro, obtendo em torno de $80 \%$ de aceitação pelos consumidores.

Palavras-chave: carne suína; transglutaminase; desossa; moldagem.

\begin{abstract}
The traditional methodologies of dry-cured ham production use the entire ham, differently from the one proposed in this study that combined boning, addition of transglutaminase, and tumbling and moulding before the drying and ageing stages. The effects of two levels of added $\mathrm{NaCl}(\mathrm{T} 1-3.5 \%$ and $\mathrm{T} 2-5 \%)$ on the physicochemical and microbiological characteristics of dry-cured hams during the process and the sensory analyses of the final products were evaluated. The dry-cured hams met the physicochemical and microbiological standards of the Brazilian legislation, and no significant differences $(\mathrm{p}<0.05)$ were found between the two treatments in the parameters evaluated during the process and in the final products, except for the weight loss, which was higher in T1 (39.74 $\pm 4.02 \%)$ than in T2 (37.22 $\pm 2.96 \%)$. The shape and thickness of the dry-cured hams prepared in this research were adequate to slicing, and they had excellent appearance, typical aroma, and flavor similar to traditional dry-cured hams usually found in the Brazilian market receiving about $80 \%$ of acceptance by consumers.
\end{abstract}

Keywords: pork; boning; transglutaminase; moulding.

\section{Introdução}

As metodologias de processamento dos presuntos crus diferem em vários aspectos desde a matéria-prima, como raça, sistema de alimentação e idade de abate dos suínos, teor de gordura, peso e grupos musculares dos pernis, até as técnicas de processamento propriamente ditas, como tipo de cura e condições/duração das etapas de repouso, secagem e maturação (ARNAU, 1998).

Dentre os principais sistemas de elaboração de presuntos crus encontram-se o Presunto Serrano (TOLDRÁ; FLORES, 1998; ARNAU; GOU, 2001), o Presunto Ibérico (CÓRDOBA et al., 1994; ANDRÉS et al., 2004), o Presunto Parma (PAROLARI, 1996; PALMIA et al., 1992) e o Presunto Francês (BUSCAILHON; BERDAGUÉ; MONIN, 1993). Todos esses utilizam pernis inteiros, com couro e ossos, e o tempo necessário para se chegar ao produto final varia entre 10 e 24 meses. Vários procedimentos como remoção dos ossos
(KEMP et al., 1980), tenderização (MARRIOT et al., 1985) e massageamento (LEAK et al., 1984; MARRIOT et al., 1987) já foram estudados para redução do tempo de fabricação. A remoção dos ossos foi o método considerado mais satisfatório para reduzir o tempo de processo sem alterar a qualidade sensorial dos presuntos crus.

A legislação brasileira (BRASIL, 2000) permite a elaboração de presuntos crus a partir de pernis inteiros ou de seus cortes. Assim, a desossa dos pernis, associada ao uso da transglutaminase (TG), uma enzima que vem sendo utilizada para reunificar músculos (MOTOKI; KUMAZAWA, 2000), poderia ser usada na fabricação de presuntos crus, visando a redução no tempo de processamento. Contudo, nenhum estudo sobre uso de TG para elaboração de presuntos crus foi encontrado na literatura disponível até o momento.

Recebido para publicação em 29/7/2008

Aceito para publicação em 16/5/2009 (003679)

${ }^{1}$ Departamento de Tecnologia de Alimentos, Universidade Estadual de Campinas - UNICAMP, Rua Monteiro Lobato, n. 80, CP 6121, CEP 13083-862, Campinas - SP, Brasil

${ }^{2}$ Instituto de Tecnologia de Alimentos, Centro de Pesquisa e Desenvolvimento de Carnes, Av. Brasil, n. 2880, CEP 13070-178, Campinas - SP, Brasil, E-mail: tfacco@ital.sp.gov.br

${ }^{*}$ A quem a correspondência deve ser enviada 
O objetivo deste estudo foi testar um novo conceito de elaboração de presuntos crus, utilizando desossa, cura individual dos principais grupos musculares do pernil suíno e reestruturação usando TG. Foram avaliados os efeitos de dois teores de $\mathrm{NaCl}$ adicionados (3,5 e 5,0\%) em parâmetros físicoquímicos e microbiológicos dos presuntos crus ao longo do processo, e em características sensoriais do produto final.

\section{Material e métodos}

\subsection{Matéria-prima}

Para a elaboração dos presuntos, foram utilizados pernis (peso médio $=14,23 \pm 0,97 \mathrm{~kg}$ ) de 32 animais provenientes de um cruzamento industrial com três raças de suínos (Landrace, Large White e Duroc), matéria-prima semelhante à utilizada na elaboração de presuntos tipo Parma.

\subsection{Descrição do processo}

Após a esfola e a desossa dos pernis, fez-se a separação de seus cinco principais cortes, os quais foram utilizados para elaboração dos presuntos crus: 1) patinho (Quadriceps femoris); 2) lagarto (Semitendinosus); 3) coxão duro (Biceps femoris e Gastrocnemius); 4) coxão mole (Semimembranosus, Adductor femoris e Gracilis); e 5) alcatra (Gluteus medius, G. accessorius e G. profundus). Foram realizados dois tratamentos variando os teores de cloreto de sódio (T1-3,5\% e T2-5\%). Além do $\mathrm{NaCl}$, foram utilizados $400 \mathrm{ppm}$ de nitrato de sódio e $200 \mathrm{ppm}$ de nitrito de sódio. Esta mistura foi distribuída manualmente sobre os cortes que, em seguida, foram transferidos para uma câmara frigorífica, onde foram mantidos sobre prateleiras vazadas de aço inoxidável, sob condições de temperatura de $2,5 \pm 1,31^{\circ} \mathrm{C}$ e umidade relativa de $83,6 \pm 2,75 \%$ por sete dias (Repouso). Após este período, fez-se a remoção do excesso de gordura externa dos cortes e a aplicação superficial de $1 \%$ de transglutaminase (Activa TG- ${ }^{\circledast}$, Ajinomoto) na forma de uma solução $25 \%$ e foi feito tumbling por 3 minutos a 7,5 rpm com a finalidade de homogeneizar a distribuição da enzima nos cortes. Para obtenção do formato desejado, fez-se a moldagem em forma de $5 \mathrm{~kg}$ para presunto cozido durante 24 horas a $4,5^{\circ} \mathrm{C}$. Em seguida, os presuntos crus foram removidos das formas, embalados a vácuo e colocados novamente nas mesmas formas por 24 horas, a fim de diminuir a espessura das peças e facilitar a equalização do sal. Transcorrido o tempo, as embalagens foram removidas e os presuntos crus foram recolocados nas prateleiras da câmara frigorífica para Secagem $\left(4,5 \pm 0,85^{\circ} \mathrm{C}\right.$ e UR de 71,62 $\pm 1,75 \%$ ) durante 21 dias. Decorrido este tempo, iniciou-se o período de Maturação. Nesta etapa, com duração de 90 dias, a temperatura da câmara foi elevada gradualmente em $\pm 1{ }^{\circ} \mathrm{C}$ a cada 10 dias. Os presuntos foram então embalados a vácuo e mantidos por 3 dias a $17^{\circ} \mathrm{C}$ na câmara fria, para equalização da umidade no produto. Em seguida, as embalagens foram retiradas e a temperatura elevada para $27^{\circ} \mathrm{C}$ por 2 dias, para acentuar os atributos sensoriais característicos de presunto cru. A temperatura foi reduzida para $15^{\circ} \mathrm{C}$ por 24 horas e, finalmente, os presuntos crus foram embalados a vácuo e armazenados sob refrigeração.

\subsection{Amostragem}

Para as análises físico-químicas e microbiológicas da matéria-prima foram coletadas três amostras. Cada amostra foi constituída de duas subamostras de pernil (direito e esquerdo do mesmo animal), de $300 \mathrm{~g}$ de cada pernil. Em cada ponto de amostragem, descritos no item 2.4 e referentes às fases do processamento, foram coletados três pares de presunto. Cada peça foi dividida ao meio, sendo uma metade destinada às análises microbiológicas e a outra às físico-químicas.

A coleta de amostra para a contagem da microbiota superficial foi realizada através da técnica do esfregaço de superfície (SILVA; JUNQUEIRA; SILVEIRA, 2001). Para a obtenção da amostra destinada à contagem da microbiota interna, desprezou-se pelo menos um centímetro de toda superfície de cada presunto, restando assim a porção interna, a qual foi homogeneizada.

Quanto às determinações físico-químicas, realizou-se primeiramente a análise de atividade de água $\left(\mathrm{a}_{\mathrm{w}}\right)$, para evitar a perda de umidade da amostra. Para a leitura da $a_{w}$ superficial, retirou-se uma fatia de aproximadamente cinco milímetros da face cárnea (superfície sem a camada de gordura) do presunto cru. Em seguida, foram retiradas três fatias verticais do produto para leitura da $a_{w}$ interna. Em cada fatia, a determinação da $a_{w}$ foi feita em três sítios, formando uma diagonal. $\mathrm{O}$ ponto próximo à face cárnea foi denominado "Zona A", o ponto central da fatia, “Zona B", e o ponto próximo à face com a camada de gordura, “Zona C”. O restante da peça foi homogeneizado para as demais análises físico-químicas.

\subsection{Análises físico-químicas e microbiológicas}

Quanto às análises físico-químicas, na preparação da matéria-prima foram realizadas análises de composição centesimal, teor de cloretos e $\mathrm{pH}$. Durante o processo, os presuntos crus foram avaliados nos dias primeiro, décimo e vigésimo de secagem, e no trigésimo e sexagésimo dia de maturação quanto a $\mathrm{a}_{\mathrm{w}}, \mathrm{pH}$, teores de cloretos e de umidade. Já no produto final foram analisados composição centesimal, teor de cloretos, teores de nitrato e nitrito, $\mathrm{pH} \mathrm{e} \mathrm{a}_{\mathrm{w}}$.

As análises de composição centesimal (teores de umidade, proteína, lipídios e resíduo mineral fixo), teor de cloretos, e teores de nitrito e nitrato foram realizadas de acordo com Instrução Normativa no 20 de 21 de julho de 1999 do Ministério da Agricultura (BRASIL, 1999). Para mensuração do pH, fez-se medida direta nos pernis (matéria-prima) e nos presuntos crus com eletrodo tipo penetração (modelo DME-CF1, DIGIMED), utilizando peagâmetro DIGIMED modelo DM-21. A a foi determinada utilizando-se um analisador AQUALAB modelo CX-2T.

Na preparação da matéria-prima, e no produto final foram realizadas as seguintes contagens microbiológicas: bolores e leveduras, mesófilos aeróbios totais, bactérias lácticas, Salmonella sp., Staphylococcus aureus, clostrídios sulfitoredutores, coliformes totais, Escherichia coli O157:H77 e Listeria monocytogenes, atendendo à legislação brasileira vigente. Durante o processo foram realizadas contagens de bolores e 
leveduras, microrganismos psicrotróficos e bactérias lácticas, no início e aos vinte dias de secagem, aos trinta e sessenta dias de maturação e ao final do processo.

As determinações de Listeria monocytogenes (ASSOCIATION OF OFFICIAL ANALYTICAL CHEMISTS, 2000), Salmonella sp. (ASSOCIATION OF OFFICIAL ANALYTICAL CHEMISTS, 2002) e Escherichia coli O157:H77 (ASSOCIATION OF OFFICIAL ANALYTICAL CHEMISTS, 1999) foram feitas pelo método da Polimerase Chain Reaction (PCR), utilizando o sistema BAX (Dupont/Qualicon). As demais determinações foram realizadas de acordo com as metodologias descritas no Compendium of Methods for the Microbiological Examination of Foods (DOWNES; ITO, 2001).

\subsection{Análise sensorial}

Um teste de localização central foi realizado com consumidores de presunto cru em um supermercado de Campinas-SP para avaliar a aceitação, preferência e intenção de compra em relação aos dois produtos elaborados. Os consumidores registraram suas notas de aceitação em relação à impressão global, aparência, aroma, sabor e textura em escala hedônica estruturada mista de 9 pontos (MEILGAARD; CIVILLE; CARR, 1999). Para a intenção de compra, foi utilizada uma escala estruturada verbal de 5 pontos, variando de "certamente compraria" a "certamente não compraria". Além dos testes de aceitação e de intenção de compra, foi realizado um teste de preferência dos consumidores em relação aos dois produtos avaliados.

\subsection{Análise estatística}

Os dados obtidos foram submetidos ao Teste T de Student, ao nível de $5 \%$ de significância, utilizando o programa SAS (SAS INSTITUTE, 1993).

\section{Resultados e discussão}

\subsection{Parâmetros físico-químicos}

A matéria-prima cárnea destinada à elaboração dos presuntos crus apresentou as seguintes características: teor de umidade de $73,48 \pm 0,95 \%$, teor de gordura de $3,49 \pm 1,02 \%$, teor de proteína de $21,93 \pm 0,88 \%$, teor de resíduo mineral fixo de $1,18 \pm 0,03 \%$, teor de cloretos de $0,13 \pm 0,02 \%$ e pH 5,81 $\pm 0,06$.

Os valores de proteína, umidade e gordura apresentados foram semelhantes aos obtidos por Virgili et al. (1998), que encontraram os seguintes valores na composição dos pernis utilizados para elaboração de presuntos Parma: proteína, 22,3\%; umidade, 73,7\%; e gordura, 3,89\%. García-Rey et al. (2004) estudaram a relação entre o $\mathrm{pH}$ antes da salga e a qualidade do presunto cru espanhol, e classificaram o $\mathrm{pH}$ da matériaprima como $\mathrm{pH}$ baixo, valores inferiores a 5,5, e $\mathrm{pH}$ normal, valores próximos de 5,8. Assim, os pernis utilizados na presente pesquisa apresentaram $\mathrm{pH}$ considerado normal.

Os resultados das análises físico-químicas dos presuntos crus maturados (Tabela 1) não apresentaram diferenças entre os tratamentos ao nível de $5 \%$ de significância, portanto, os dois teores de sal inicialmente adicionados (3,5 e 5\%) não resultaram em diferenças nas características físico-químicas do produto final. Durante a salga, nem todo o sal adicionado foi incorporado na carne. Parte foi perdida nas bandejas de salga ou depois, através das prateleiras vazadas na câmara fria. Como no T2 mais sal foi adicionado, também mais sal foi perdido, o que provavelmente resultou na diferença não significativa de composição entre os tratamentos. O teor de cloretos nos presuntos após o período de salga, no dia 10 do processo, foi de 3,07 $\pm 0,23$ e de $3,21 \pm 0,35$ ( $p>0,05)$.

O sal promove maior solubilização proteica, aumentando a capacidade de retenção de água por capilaridade (OFFER; TRINICK, 1983). O valor mínimo para que a diferença entre os teores de umidade dos tratamentos fosse considerada significativa $(\mathrm{p}<0,05)$ foi de 5,85 e a diferença observada foi de 4,39, sendo que T2 apresentou valor mais elevado $(53,05 \pm 2,38)$ em relação a T1 $(48,65 \pm 2,76)$, provavelmente devido à maior quantidade de sal incorporada no T2, embora o teor de cloretos não tenha apresentado diferença significativa entre os tratamentos.

Os dados apresentados atendem aos requisitos da legislação brasileira sobre os padrões de identidade e qualidade de presuntos crus (BRASIL, 2000). Segundo os regulamentos técnicos, produtos classificados como "Presuntos Crus" devem possuir no máximo $20 \%$ de gordura e no mínimo $27 \%$ de proteínas. Enquanto para ser considerado um presunto "Tipo Parma” ou "Tipo Serrano", o produto deve possuir no máximo 15\% de gordura e no mínimo $27 \%$ de proteína.

Os resultados obtidos para os teores de nitrato e nitrito atendem aos limites estipulados pela legislação brasileira, cuja soma dos dois deve ser inferior a $300 \mathrm{mg} \cdot \mathrm{kg}^{-1}$ de produto. Nos processos em que se usam somente nitratos, os teores de nitritos aumentam somente durante a fase de repouso e no início da secagem, especialmente nas zonas superficiais e praticamente desaparecem até o final desta última (ARNAU et al., 1995)

Toscani et al. (2000) estudaram os efeitos de três processos de fabricação (três diferentes fábricas), nas composições de presuntos Parma após 13 meses de processo e obtiveram diferenças nos parâmetros avaliados entre si. Os resultados variaram de 24,7 a $26,8 \%$ para proteína, de 5,3 a $6,9 \%$ para gordura, e de 59,5 a $62,9 \%$ para umidade.

Tabela 1. Características físico-químicas dos presuntos crus ao final do processo*.

\begin{tabular}{lrr}
\hline \multicolumn{1}{c}{ Parâmetro } & \multicolumn{1}{c}{ T1 } & \multicolumn{1}{c}{ T2 } \\
\hline Umidade (\%) & $48,65 \pm 2,76$ & $53,04 \pm 2,37$ \\
Gordura (\%) & $6,73 \pm 3,45$ & $4,70 \pm 1,44$ \\
Proteína (\%) & $36,66 \pm 2,24$ & $34,58 \pm 1,84$ \\
Resíduo mineral fixo (\%) & $7,14 \pm 0,79$ & $7,83 \pm 0,75$ \\
Cloretos (\%) & $5,53 \pm 1,03$ & $6,26 \pm 0,82$ \\
Nitrito de sódio (mg.kg $\left.{ }^{-1}\right)$ & $15,07 \pm 4,44$ & $21,85 \pm 8,51$ \\
Nitrato de sódio (mg.kg ${ }^{-1}$ ) & $258,70 \pm 15,35$ & $224,60 \pm 28,76$ \\
\hline${ }^{*}$ Para todos os parâmetros, as diferenças não foram significativas ao nível de 5\% de \\
significância.
\end{tabular}


Palmia et al. (1992), analisando as características físicoquímicas dos músculos Semimembranosus e Biceps femoris de presuntos de Parma, observaram diferenças nos teores de cloretos, proteína e umidade entre os músculos. No músculo Semimembranosus, os valores de proteína variaram de 29,17 a 33,58\%, entre 5,57 e 6,41\% para cloretos e entre 48,64 e $51,54 \%$ para umidade. Já no músculo Biceps femoris, os valores de proteína variaram entre 28,40 e 31,95\%, de 6,65 a 7,62\% para cloretos e entre 53,15 e $55,95 \%$ para umidade.

Sánchez (2003) obteve teores de sal de 6,09 e 6,03\% em presuntos crus elaborados sem e com cultura starter, respectivamente. Já Arnau et al. (1995), analisando pernis não desossados, obtiveram aos 122 dias de processo os seguintes valores de $\mathrm{NaCl}$ nos respectivos músculos: Semimembranosus, 6,9\%; Gracilis, 2,9\%; Biceps femoris, 5,9\%; Semitendinosus, 4,2\%; Gastrocnemius, $4 \%$.

Apesar da composição dos produtos obtidos neste trabalho se enquadrarem nos requisitos da legislação brasileira de classificação de presunto cru, os valores de alguns parâmetros diferem da literatura internacional, pois as metodologias de fabricação apresentam diferenças desde o preparo da matériaprima, tempo de processo, até a forma de apresentação do produto final.

Não foram encontradas diferenças significativas nos teores de umidade e de cloretos, e de $\mathrm{pH}$ entre os tratamentos $(\mathrm{p}<0,05)$, em todos os pontos de amostragem no decorrer do processo (Tabela 2).

Durante o processamento de presuntos crus, ocorre perda de umidade e, como consequência, há aumento da concentração dos outros compostos, alterando as características físicoquímicas e a composição centesimal dos produtos.

Arnau et al. (1995) concluíram que a distribuição de sal no pernil depende principalmente da distribuição de água, e que a concentração de salmoura tende a se equalizar nos músculos mais importantes com o decorrer do processo. De acordo com os autores, a concentração de sal do músculo Semimembranosus diminui durante a maturação, já que este segue em direção aos músculos mais úmidos, localizados mais internamente no pernil íntegro. Monin et al. (1997) observaram a mesma tendência de diminuição da concentração salina no músculo Semimembranosus, e aumento no músculo Semitendinosus, assim como no músculo Rectus femoris. A velocidade desta equalização pode ser reduzida pela presença de gordura ou de tecido conjuntivo ou de ossos, que podem atuar como barreiras físicas (ARNAU et al., 1995).

García Rey et al. (2004) verificaram que o pH do presunto se relaciona com o $\mathrm{pH}$ da matéria-prima, que pernis com $\mathrm{pH}$ baixo $(5,41)$ dão origem a presuntos com $\mathrm{pH}$ baixo $(5,86)$, e pernis com $\mathrm{pH}$ normal $(5,79)$ originam produtos com $\mathrm{pH}$ normal $(6,04)$. O pH final dos presuntos crus nos processos tradicionais $(>6,0)$ é atribuído às reações proteolíticas, como consequência da hidrólise de aminoácidos e da exposição de grupos básicos. Essa tendência não foi constatada no presente estudo, no qual, a partir de pernis com $\mathrm{pH}$ normal, foram obtidos produtos com $\mathrm{pH}$ mais baixo $(\sim 5,60)$. O curto período de maturação e as temperaturas abaixo dos $16^{\circ} \mathrm{C}$ durante quase todo o processo podem ter retardado a ação enzimática, não levando ao aumento do $\mathrm{pH}$. A velocidade da proteólise também pode ter sido reduzida pela ação do sal, já que os cortes cárneos foram salgados individualmente, levando a uma distribuição de sal mais uniforme nas peças, o que não acontece nos processos tradicionais, nos quais o sal demora a se difundir até as porções mais internas do pernil. Ao contrário do que normalmente é observado nos produtos tradicionais, neste estudo, o $\mathrm{pH}$ diminuiu ao longo do processo, o que provavelmente se deve à ação das bactérias lácticas, cujas contagens atingiram de 1,13 a $1,70 \times 10^{6}$ UFC. $\mathrm{g}^{-1}$ nos presuntos maturados de T1 e T2, respectivamente (Tabela 5).

Os pHs finais dos presuntos crus dos tratamentos T1 e T2 foram 5,60 e 5,56, respectivamente. Arnau et al. (1995) estudaram mudanças no $\mathrm{pH}$ em diferentes músculos de presuntos normais e PSE durante o processamento, como Vastus medialis, V. lateralis, V. intermedius, Rectus femoris, Gastrocnemius, Semitendinosus, Biceps femoris, Semimembranosus, Gracilis e Gluteus. De todos os valores finais de $\mathrm{pH}$ destes músculos após 207 dias de processo, o menor foi 6,2 nos músculos Semitendinosus, Semimembranosus e Biceps femoris.

Sánchez (2003) estudou os efeitos do uso de cultura iniciadora e da aplicação de gordura fundida sobre os presuntos crus para evitar a perda excessiva de umidade durante a maturação, e obteve pHs ao redor de 5,7 nos músculos Semimembranosus dos presuntos crus, o que foi atribuído ao menor grau de proteólise causado pela secagem excessiva na superfície.

Nos processos tradicionais de fabricação de presunto Serrano, somente partes do couro e da gordura subcutânea são

Tabela 2. Valores de $\mathrm{pH}$ e teores de umidade e cloretos dos presuntos crus durante o processo*.

\begin{tabular}{|c|c|c|c|c|c|c|}
\hline \multirow{3}{*}{$\begin{array}{l}\text { Tempo } \\
\text { (Dias) }\end{array}$} & \multicolumn{6}{|c|}{ Tratamentos } \\
\hline & $\mathrm{T} 1$ & $\mathrm{~T} 2$ & $\mathrm{~T} 1$ & $\mathrm{~T} 2$ & $\mathrm{~T} 1$ & $\mathrm{~T} 2$ \\
\hline & \multicolumn{2}{|c|}{ Umidade (\%) } & \multicolumn{2}{|c|}{ Cloretos (\%) } & \multicolumn{2}{|c|}{$\mathrm{pH}$} \\
\hline 10 & $70,27 \pm 0,71$ & $71,72 \pm 1,55$ & $3,07 \pm 0,23$ & $3,21 \pm 0,35$ & $5,92 \pm 0,20$ & $5,69 \pm 0,05$ \\
\hline 20 & $67,42 \pm 0,56$ & $66,71 \pm 0,90$ & $3,46 \pm 0,54$ & $4,02 \pm 0,24$ & $6,07 \pm 0,08$ & $6,02 \pm 0,07$ \\
\hline 30 & $62,74 \pm 2,79$ & $63,89 \pm 2,96$ & $3,99 \pm 0,30$ & $3,98 \pm 1,00$ & $5,92 \pm 0,16$ & $5,88 \pm 0,14$ \\
\hline 61 & $59,69 \pm 0,88$ & $59,19 \pm 2,24$ & $4,15 \pm 0,55$ & $4,33 \pm 0,77$ & $5,98 \pm 0,09$ & $5,92 \pm 0,08$ \\
\hline 91 & $52,29 \pm 3,47$ & $53,27 \pm 1,80$ & $4,51 \pm 0,34$ & $5,62 \pm 0,54$ & $5,59 \pm 0,17$ & $5,51 \pm 0,04$ \\
\hline 125 & $48,65 \pm 2,76$ & $53,05 \pm 2,38$ & $5,53 \pm 1,03$ & $6,26 \pm 0,82$ & $5,60 \pm 0,12$ & $5,56 \pm 0,08$ \\
\hline
\end{tabular}

${ }^{\star}$ Para todos os parâmetros, as diferenças não foram significativas ao nível de $5 \%$ de significância. 
removidas do pernil (ARNAU; GOU, 2001), o que não aconteceu no processo acelerado utilizado neste trabalho, em que todo o couro e parte da gordura subcutânea foram retirados. A ausência destes favoreceu a secagem e a redução da $a_{w}$ para os valores finais desejados. Os valores finais de $a_{w}$ obtidos em ambos os tratamentos, atendem aos requisitos da legislação brasileira sobre os padrões de identidade e qualidade de presuntos crus (BRASIL, 2000), os quais devem ser inferiores a 0,92.

No decorrer do processo há concentração do teor de $\mathrm{NaCl}$ no produto devido à redução do teor de umidade decorrente das perdas por evaporação. Com o aumento da concentração salina, há redução dos valores de $\mathrm{a}_{\mathrm{w}}$ (Tabela 3). A absorção de sal e a desidratação são consideradas as principais responsáveis pela estabilidade dos presuntos crus (GOU; COMAPOSADA; ARNAU, 2004), pois ambas contribuem para a $\mathrm{a}_{\mathrm{w}}$ final do produto.

Os valores de $\mathrm{a}_{\mathrm{w}}$ não foram homogêneos nas diferentes zonas de amostragem dos produtos durante o processamento, com o centro das peças (zona B) tendendo a apresentar os valores mais elevados. Só ao final do processo, quando os presuntos crus foram embalados a vácuo, a umidade e os valores de $\mathrm{a}_{\mathrm{w}}$ se tornaram uniformes em toda a peça.

Com relação à perda de peso acumulada ao final do processo (Tabela 4), observa-se que T1 apresenta um valor significativamente $(\mathrm{p}<0,05)$ superior a T2. Em baixas concentrações, como as utilizadas neste trabalho, o sal faz com que a carne retenha a maior quantidade de água (OFFER; TRINICK, 1983) e, como a concentração final de sal do tratamento T2 foi levemente superior, isso pode ter acarretado em maior retenção de água e menores perdas de peso nestes presuntos crus.

Os valores de perda de peso ao final do processo foram similares aos encontrados em outros trabalhos, apesar do tempo de processamento ser menor. Para Barroso e López (2001), a perda de peso média do presunto Ibérico ao final do processo foi de 32\% e Sánchez (2003) obteve valores de 36,7\% em presuntos Serranos elaborados com cultura iniciadora. Já Palmia et al. (1992), avaliando as perdas de peso durante todo o processamento de presuntos crus, obtiveram os seguintes resultados: $4,82 \%$ aos
27 dias; 9,44\% aos 55 dias; 14,89\% aos 96 dias; e 28,38\% aos 392 dias (final do processo).

\subsection{Análises microbiológicas}

Os resultados obtidos quanto ao perfil microbiológico da matéria-prima e dos produtos finais (Tabela 5) mostram que ela se encontrava de acordo com os padrões microbiológicos para o produto estudado, segundo RDC no 12 de 2001 da Agência Nacional da Vigilância Sanitária (BRASIL, 2001). Não foi verificada a presença de patógenos importantes para a saúde pública, como as bactérias Clostridium perfringens; E. coli O157:H7 e de Listeria monocytogenes.

As principais barreiras utilizadas no processamento (d, temperatura, nitrificantes) foram eficazes na manutenção da segurança microbiológica do produto para o consumidor. Inclusive a contagem de psicrotróficos, que incluem microrganismos deteriorantes de alimentos refrigerados, ficou no nível de $10^{3}$ UFC.g ${ }^{-1}$, considerado baixo (SILVA; JUNQUEIRA; SILVEIRA, 2001). A população de fungos encontrada constituise basicamente de leveduras. Estas têm temperatura ótima de crescimento em torno de $25^{\circ} \mathrm{C}$, podendo se desenvolver sob temperaturas de refrigeração. Conforme esperado, a contagem final de fungos ficou no nível de $10^{4}$ a $10^{5}$ UFC.g $^{-1}$, mesmos valores encontrados por Núñez et al. (1996). As contagens de cocos gram-positivos, bactérias lácticas e fungos algumas vezes aumentam durante o processamento de presuntos crus, como foi observado no presente trabalho, porque esses microrganismos são halotolerantes (KEMP et al., 1980).

\subsection{Análise sensorial}

O teste de localização central contou com a participação de 110 pessoas. Comparando os dois produtos (T1 e T2), com relação à aceitação global, aparência, aroma, sabor e textura, verificou-se que não houve diferença $(\mathrm{p}<0,05)$ entre os produtos em nenhum dos aspectos questionados, sendo que, para ambos, cerca de $80 \%$ dos consumidores gostaram dos produtos de maneira geral. $\mathrm{O}$ teste de preferência dos consumidores também não detectou diferença significativa entre os produtos,

Tabela 3. Valores de Atividade de água no presunto cru durante o processo.

\begin{tabular}{|c|c|c|c|c|c|}
\hline \multirow{2}{*}{$\begin{array}{l}\text { Tempo } \\
\text { (Dias) }\end{array}$} & \multirow{2}{*}{ Tratamento } & \multicolumn{4}{|c|}{ Zona de amostragem } \\
\hline & & $\mathrm{S}$ & A & $\mathrm{B}$ & $\mathrm{C}$ \\
\hline \multirow[t]{2}{*}{10} & $\mathrm{~T} 1$ & $0,973 \pm 0,005^{\mathrm{a}}$ & $0,970 \pm 0,004^{\mathrm{a}}$ & $0,976 \pm 0,002^{\mathrm{a}}$ & $0,975 \pm 0,007^{\mathrm{a}}$ \\
\hline & $\mathrm{T} 2$ & $0,975 \pm 0,005^{\mathrm{ab}}$ & $0,971 \pm 0,004^{\mathrm{b}}$ & $0,978 \pm 0,005^{\mathrm{a}}$ & $0,969 \pm 0,007^{\mathrm{b}}$ \\
\hline \multirow[t]{2}{*}{20} & $\mathrm{~T} 1$ & $0,957 \pm 0,010^{\mathrm{b}}$ & $0,963 \pm 0,009^{\mathrm{ab}}$ & $0,971 \pm 0,008^{\mathrm{a}}$ & $0,964 \pm 0,007^{\mathrm{ab}}$ \\
\hline & $\mathrm{T} 2$ & $0,948 \pm 0,007^{c}$ & $0,955 \pm 0,008^{b c}$ & $0,966 \pm 0,006^{\mathrm{a}}$ & $0,960 \pm 0,011^{\mathrm{ab}}$ \\
\hline \multirow[t]{2}{*}{30} & $\mathrm{~T} 1$ & $0,941 \pm 0,002^{c}$ & $0,957 \pm 0,007^{\mathrm{b}}$ & $0,964 \pm 0,003^{\mathrm{a}}$ & $0,959 \pm 0,004^{\mathrm{ab}}$ \\
\hline & $\mathrm{T} 2$ & $0,931 \pm 0,009^{\mathrm{b}}$ & $0,951 \pm 0,007^{\mathrm{a}}$ & $0,960 \pm 0,009^{\mathrm{a}}$ & $0,956 \pm 0,010^{\mathrm{a}}$ \\
\hline \multirow[t]{2}{*}{61} & $\mathrm{~T} 1$ & $0,941 \pm 0,003^{c}$ & $0,947 \pm 0,005^{\mathrm{b}}$ & $0,954 \pm 0,002^{\mathrm{a}}$ & $0,943 \pm 0,002^{\mathrm{bc}}$ \\
\hline & $\mathrm{T} 2$ & $0,939 \pm 0,007^{\mathrm{b}}$ & $0,945 \pm 0,009^{\mathrm{ab}}$ & $0,950 \pm 0,007^{\mathrm{a}}$ & $0,940 \pm 0,005^{\mathrm{b}}$ \\
\hline \multirow[t]{2}{*}{91} & $\mathrm{~T} 1$ & $0,912 \pm 0,022^{b}$ & $0,929 \pm 0,011^{\mathrm{ab}}$ & $0,937 \pm 0,011^{\mathrm{a}}$ & $0,930 \pm 0,011^{\mathrm{ab}}$ \\
\hline & $\mathrm{T} 2$ & $0,904 \pm 0,017^{\mathrm{b}}$ & $0,921 \pm 0,005^{\mathrm{a}}$ & $0,926 \pm 0,006^{\mathrm{a}}$ & $0,920 \pm 0,010^{\mathrm{a}}$ \\
\hline \multirow[t]{2}{*}{125} & $\mathrm{~T} 1$ & $0,888 \pm 0,017^{\mathrm{a}}$ & $0,887 \pm 0,017^{\mathrm{a}}$ & $0,900 \pm 0,017^{\mathrm{a}}$ & $0,887 \pm 0,019^{\mathrm{a}}$ \\
\hline & $\mathrm{T} 2$ & $0,899 \pm 0,010^{\mathrm{a}}$ & $0,893 \pm 0,017^{\mathrm{a}}$ & $0,905 \pm 0,012^{\mathrm{a}}$ & $0,897 \pm 0,014^{\mathrm{a}}$ \\
\hline
\end{tabular}

${ }^{\mathrm{abc}}$ Médias com letras iguais na mesma linha não diferem estatisticamente $(\mathrm{p}<0,05)$. 
Tabela 4. Perda de peso acumulada durante os processos.

\begin{tabular}{ccr}
\hline \multirow{2}{*}{$\begin{array}{c}\text { Tempo } \\
\text { (Dias) }\end{array}$} & \multicolumn{2}{c}{ Perda de peso acumulada (\%) } \\
\cline { 2 - 3 } & \multicolumn{1}{c}{ T1 } & \multicolumn{1}{c}{ T2 } \\
\hline 17 & $4,90 \pm 1,21^{\mathrm{a}}$ & $4,60 \pm 0,96^{\mathrm{a}}$ \\
24 & $13,15 \pm 1,56^{\mathrm{a}}$ & $12,07 \pm 1,40^{\mathrm{b}}$ \\
31 & $19,15 \pm 2,23^{\mathrm{a}}$ & $17,70 \pm 1,91^{\mathrm{a}}$ \\
38 & $23,69 \pm 2,70^{\mathrm{a}}$ & $22,31 \pm 1,99^{\mathrm{a}}$ \\
125 & $39,74 \pm 4,02^{\mathrm{a}}$ & $37,22 \pm 2,96^{\mathrm{b}}$ \\
\hline
\end{tabular}

${ }^{\mathrm{ab}}$ Médias com letras iguais na mesma linha não diferem estatisticamente $(\mathrm{p}<0,05)$.

Tabela 5. Perfil microbiológico da matéria-prima e dos produtos finais.

\begin{tabular}{llll}
\hline \multicolumn{1}{c}{ Determinações } & Matéria-prima & T1 & T2 \\
\hline Bactérias Láticas * $^{*}$ & $2,8 \times 10^{5}$ & $1,13 \times 10^{6}$ & $1,70 \times 10^{6}$ \\
Fungos $^{*}$ & $1,7 \times 10^{1}$ & $3,80 \times 10^{5}$ & $2,42 \times 10^{5}$ \\
C. perfringens ${ }^{*}$ & $<10$ & $<10$ & $<10$ \\
Coliformes Fecais & $<10$ & $<10$ & $<10$ \\
Listeria monocytogenes & ausência & ausência & ausência \\
(em 25g) & & & \\
Salmonella sp. & ausência & ausência & ausência \\
(em 25 g) & & & \\
Contagem total de Mesófilas * & $1,0 \times 10^{4}$ & $2,69 \times 10^{5}$ & $1,91 \times 10^{5}$ \\
Contagem total de Psicrotróficos & $<10$ & $1,30 \times 10^{3}$ & $3,00 \times 10^{3}$ \\
S. aureus ${ }^{*}$ & $<10^{2}$ & $<10^{2}$ & $<10^{2}$ \\
E. coli O157:H7 (em 25 g) & ausência & ausência & ausência \\
\hline
\end{tabular}

*Unidades formadoras de colônias por grama.

confirmando a boa aceitação de ambos. A intenção de compra dos presuntos crus refletiu os resultados dos testes de aceitação e de preferência. A maioria dos consumidores provavelmente ou certamente compraria os dois produtos.

\section{Conclusões}

A remoção de barreiras físicas à distribuição do sal acarretou a redução do tempo das etapas de salga e equalização dos presuntos, diminuindo assim o tempo total do processo para 5 meses. O processo acelerado de elaboração de presunto cru desenvolvido permitiu a obtenção de presunto cru microbiologicamente estável e com características físicoquímicas desejáveis. Apesar do curto tempo de maturação (cerca de 95 dias), os presuntos apresentaram gosto e aroma característicos de presunto cru, semelhantes aos de produtos tradicionalmente produzidos.

\section{Agradecimentos}

Os autores agradecem à FAPESP o financiamento do Projeto (02/10889-0) e ao CNPq as bolsas de Mestrado.

\section{Referências bibliográficas}

ANDRÉS, A. I. et al. Sensory characteristics of Iberian ham: influence of salt content and processing conditions. Meat Science, v. 68, p. 45-51, 2004.
ANDRÉS, A. I. et al. Sensory characteristics of Iberian ham: Influence of salt content and processing conditions. Meat Science, v. 68, p. 45-51, 2004.

ARNAU, J. et al. Physical and chemical changes in different zones of normal and PSE dry-cured ham during processing. Food Chemistry, v. 52, p. 63-69, 1995.

ARNAU, J. Tecnología del jamón curado en distintos países. In: SIMPOSIO ESPECIAL - INTERNATIONAL CONGRESS OF MEAT SCIENCE AND TECHNOLOGY, 44., 1998, Barcelona. Estrategias Alimentaris... Madrid: EUROCARNE, 1998. p. 10-21.

ARNAU, J.; GOU, P. Effect of air relative humidity on ham rind and subcutaneous salted fat during the resting period. Meat Science, v. 58 , p. 65-68, 2001.

ASSOCIATION OF OFFICIAL ANALYTICAL CHEMISTS - AOAC. BAX for screening Listeria monocytogenes: certification report of AOAC Research Institute : performance tested method 000702. 2000. Disponível em: <http://aoac.org/RI/000702.htm>. Acesso em: 20 ago. 2003.

ASSOCIATION OF OFFICIAL ANALYTICAL CHEMISTS - AOAC. Official methods of analysis. 16 ed. Gaithersburg, 1998.

ASSOCIATION OF OFFICIAL ANALYTICAL CHEMISTS AOAC. Qualicon bax system for Salmonella: certification report of AOAC Research Institute : performance tested method 100201. 2002. Disponível em: <http://www.aoac.org/ testkits/100201Salmonella\%20Report.pdf>. Acesso em: 28 abr. 2003.

BARROSO, J. V.; LÓPEZ, R. C. Dinámica y control del proceso de secado del jamón Ibérico en secaderos y bodegas naturales y en cámaras climatizadas. In: BARROSO, J. V (Ed.). Tecnología del Jamón Ibérico: de los sistemas tradicionales a la explotación racional del sabor y el aroma. Madri: Ed. Mundi Prensa, 2001. p. 255-292.

BRASIL. Ministério da Agricultura, Pecuária e Abastecimento. Aprova os regulamentos técnicos de identidade e qualidade de copa, de jerked beef, de presunto tipo Parma, de presunto cru, de salame, de salaminho, de salame tipo alemão, de salame tipo calabrês, de salame tipo friolano, de salame tipo napolitano, de salame tipo hamburguês, de salame tipo italiano, de salame tipo milano, de lingüiça colonial e pepperoni. Instrução Normativa n²2, 31 de julho de 2000. Diário Oficial. Brasília, 2000. p. 17-18.

BRASIL. Ministério da Agricultura, Pecuária e Abastecimento. Oficializa os métodos analíticos físico-químicos, para controle de produtos cárneos e seus ingredientes - sal e salmoura. Instrução Normativa $n^{\circ}$ 20, 21 de julho de 1999. Diário Oficial. Disponível em: <www.agricultura.gov.br/sda/dipoa/legislacaoprodutocarneos. htm>. Acesso em: 19 ago. 2003.

BRASIL. Ministério da Saúde. Agência Nacional de Vigilância Sanitária - ANVISA. Aprova o Regulamento Técnico sobre padrões microbiológicos para alimentos. Resolução RDC n ${ }^{\circ}, 12$ de 02 de janeiro de 2001. Diário Oficial. Disponível em: <http://e-legis.bvs. br/leisref/public/showAct.php?id=144>. Acesso em: 17 set. 2004 .

BUSCAILHON, S.; BERDAGUÉ, J. L, MONIN, G. Time-related changes in volatile compounds of lean tissue during processing of French dry-cured ham. Journal of the Science of Food and Agriculture, v. 63, p. 69-75, 1993.

CÓRDOBA, J. J. et al. Evolution of free amino acids and amines during ripening of Iberian cured ham. Journal of Agricultural and Food Chemistry, v. 42, p. 2296-2301, 1994.

DOWNES, F. P.; ITO, K. (Eds.). Compendium of methods for the microbiological examination of foods. 4 ed. Washington, D.C.: American Public Health Association, 2001. 
GARCÍA-REY, R. M. et al. Relationship between $\mathrm{pH}$ before salting and dry-cured ham quality. Meat Science, v. 67, p. 625-632, 2004.

GOU, P.; COMAPOSADA, J.; ARNAU, J. Moisture diffusivity in the lean tissue of dry-cured ham at different process times. Meat Science, v. 67, p. 203-209, 2004.

KEMP, J. D. et al. Effect of curing ingredients, skinning and boning on yield, quality and microflora of country hams. Journal of Food Science, v. 45, p. 174-177, 1980.

LEAK, F. W. et al. Effect of tumbling and tumbling time on quality and microflora of dry-cured hams. Journal of Food Science, v. 49, p. $695,1984$.

MARRIOT, N. G. et al. Accelerated dry curing of hams. Meat Science, v. 15, p. $51,1985$.

MARRIOT, N. G. et al. Accelerated production of dry-cured hams. Meat Science, v. 19, p. 53, 1987.

MEILGAARD, M.; CIVILLE, V.; CARR, B. T. Sensory evaluation techniques. 3 ed. Boca Raton: CRC Press, 1999. 387 p.

MONIN, G. et al. Chemical and structural changes in dry-cured hams (Bayonne Hams) during processing and effects of the dehairing technique. Meat Science, v.47, p. 29-47, 1997.

MOTOKI, M.; KUMAZAWA, Y. Review: recent research trends in transglutaminase technology for food processing. Food Science and Technology Research, v. 6, p. 151-160, 2000.

NÚNEZ, F. et al. Yeast population during ripening of dry-cured Iberian ham. International Journal of Food Microbiology, v. 29, p. 185-197, 1996.
OFFER, G.; TRINICK, J. On the mechanism of water holding in meat: the swelling and shrinking of myofibrils. Meat Science, v. 8, p. 245-281, 1983.

PALMIA, F. et al. Salt and water distribution in typical Italian hams. Revista Española de Ciencia y Tecnología de Alimentos, v. 32, p. 71-83, 1992.

PAROLARI, G. Review: achievements, needs and perspectives in drycured ham technology: the example of Parma ham. Food Science and Technology International, v. 2, p. 69-78, 1996.

SÁNCHEZ, F. Modificaciones tecnológicas para mejorar la seguridad y calidad del jamón curado. 2003. Tese (Doutorado) - Universitat de Girona, 2003.

SAS BUSINESS ANALYTICS SOFTWARE. SAS user's guide: statistics. Cary, 1993.

SILVA, N.; JUNQUEIRA, V. C. A.; SILVEIRA, N. F. A. Manual de métodos de análise microbiológica de alimentos. São Paulo: Editora Varela, 2001.

TOLDRÁ, F.; FLORES, M. The role of muscle proteases and lipases in flavor development during the processing of dry-cured ham. Critical Reviews in Food Science, v. 38, p. 331-352, 1998.

TOSCANI, T. et al. Effects of different processing plants on proximate composition, proteolysis and hardness of Parma ham. Industria Conserve, v. 75, n. 3, p. 259-270, 2000.

VIRGILI, R. et al. Proteases in fresh pork muscle and their influence on bitter taste formation in dry-cured ham. Journal of Food Biochemistry, v. 22, p. 53-63, 1998. 